\title{
Maximum mass-loss rates of line-driven winds of massive stars
}

\author{
C. Aerts ${ }^{1}$ and H. J. G. L. M. Lamers ${ }^{2,3}$ \\ 1 Instituut voor Sterrenkunde, Katholieke Universiteit Leuven, Celestijnenlaan 200 B, 3001 Leuven, Belgium \\ 2 Astronomical Institute, Utrecht University, PO Box 80000, 3508 TA Utrecht, The Netherlands \\ 3 SRON Laboratory, for Space Research, Sorbonnelaan 2, 3584 CA Utrecht, The Netherlands
}

Received 20 December 2002 / Accepted 3 March 2003

\begin{abstract}
We develop a theoretical treatment that allows us to determine the maximum mass-loss rate of a hot rotating star with a wind that is accelerated by radiation pressure due to spectral lines, taking into account finite disk correction as well as the effect of photon tiring but neglecting multiple scattering. The maximum mass-loss rate of a star is obtained by subsequent numerical integrations of the momentum equation from an assumed position of the sonic point onwards for increasing values of the mass loss, until the wind can no longer escape. For stars rotating below $80 \%$ of the critical velocity the decrease in the velocity far out in the wind due to the maximisation of the mass loss is negligible. Stars rotating at $>80 \%$ of the critical speed have a kinked velocity law connected with the highest possible mass-loss rate. In such cases the wind velocity increases up to typically a few stellar radii, and decreases subsequently almost ballistically outwards. In these cases the terminal wind velocity is much smaller than the maximum wind velocity. For O-type main-sequence stars, the maximum mass-loss rates derived from our formalism are somewhat smaller than those derived for self-regulated line-driven winds including multiple scattering. For B-type supergiants, however, the maximum mass-loss rate is higher by about a factor 1.5-2. Including rotation, but without gravity darkening, results in a maximum mass-loss rate that is twice as high as for a non-rotating star.
\end{abstract}

Key words. stars: early-type - stars: mass-loss - stars: winds, outflows - stars: evolution

\section{Introduction}

The subject of this paper is to derive theoretical upper limits for the mass-loss rates $\dot{M}$ that can be expelled from non-rotating and rotating massive stars due to radiation pressure in spectral lines. This upper limit is important, because evolutionary tracks of rotating massive stars show that the stars may reach a critical limit, the so-called $\Omega \Gamma$-limit, after or during their main sequence phase. This $\Omega \Gamma$-limit is the Eddington-limit modified by rotation, which is the same as the critical rotation limit, modified by radiation pressure. Evolutionary calculations of e.g. Langer (1998), Heger et al. (2000) and Maeder \& Meynet (2000a,b) show that stars at the $\Omega \Gamma$-limit have to eject a large amount of mass in a relatively short time. The question that we want to answer is: can such a high amount of mass be expelled from the star by radiation pressure in spectral lines?

The theory of a radiation-driven stellar wind in a hot nonrotating star was developed with success for the first time by Castor et al. (CAK) in a pioneering paper published in 1975. CAK used the Sobolov approximation to parametrize the radiative acceleration by large numbers of spectral lines as a simple powerlaw of an optical depth parameter. With this parametrisation they were able to solve the momentum equation that describes the hydrodynamics of an isothermal stellar wind. The CAK-theory forms the basis of many more sophisticated studies of radiation-driven winds by several different authors

Send offprint requests to: C. Aerts,

e-mail: conny@ster.kuleuven.ac.be up to the present day. Pauldrach et al. (1986) have shown the CAK-parametrisation to be very appropriate, by comparing it with the actual line driving due to a huge number of spectral lines.

Vink et al. (2000, 2001) have recently provided new theoretical mass-loss rates of $\mathrm{O}$ and $\mathrm{B}$ stars based on generalisations of the CAK-theory. They succeeded for the first time to find overall good agreement between theoretical calculations and observations of $\dot{M}$ for a wide range of effective temperatures, $12000<T_{\text {eff }}<50000 \mathrm{~K}$, by taking into account the effects of multiple scattering and different metallicities. These results are nowadays used for the calculation of evolutionary tracks of massive stars. In all these models radiation pressure on spectral lines determines both the mass-loss rate and the acceleration of the wind.

In the current paper we take a different approach: we investigate what the highest mass-loss rate is that can be accelerated and removed out of the gravitational potential well of the star by means of radiation pressure on spectral lines. In our calculations the mass-loss rate is a free parameter that may be set by some mechanism other than radiation pressure. This method is analogous to the mass-loss studies of dust-driven winds, where the acceleration is due to radiation pressure on dust, but the mass-loss rate is due to the effect of the pulsations, e.g. Wilson \& Bowen (1984) and Lamers \& Cassinelli (1999, Chap. 7). The mass-loss rates of hot stars have hardly been studied from this viewpoint. 
In our work, we investigate to what extent the conservation of momentum and energy allow the occurrence of extremely high mass loss. In order to do so, we calculate the maximum mass loss by means of a different approach compared to the classical derivation of $\dot{M}$ in the framework of radiation-driven wind theory. Traditionally, $\dot{M}$ is derived from the momentum equation, to which specific conditions - the so-called regularity and singularity condition - are added in order to find one unique value for the mass loss of an isothermal wind. Here, we do not make use of such specific conditions but we rather calculate the upper limit to the mass loss that an optically-thin stellar wind with a temperature gradient can drive, i.e. we investigate how large $\dot{M}$ can become such that the wind velocity fulfills the momentum equation and remains positive at all distances, although its gradient may become negative. We do this by simply expressing conservation of momentum and energy.

Poe et al. (1990) were the first to discuss mass-loss rates that exceed the CAK value at the expense of a non-monotonic wind velocity that declines to small terminal values (see their Fig. 4). They have found such solutions by studying in detail the steady state solution topology of line-driven winds without relying on the Sobolev approximation for the calculation of the line force. Owocki et al. (1994) performed two-dimensional hydrodynamical calculations to simulate a radiation-driven stellar wind for a rapidly rotating Be star, neglecting gravity darkening and non-radial line forces. These authors find an increase in mass-loss with a factor of about 2.8 compared to a non-rotating star when the rotation speed amounts to $80 \%$ of the critical velocity (see their Table 1). Moreover, they find the line-driving to be inadequate in the dense equatorial region, resulting in a stagnation and even reaccretion of wind material. Gayley (2000) has also considered the case where the mass loss is treated as an external parameter instead of being selfregulated by the wind, i.e. instead of being determined by the critical-point conditions of CAK theory. He proposes a scaling law for the wind velocity in the simplification of neglecting the gravity far out in the wind, which is in general a valid approximation in the situation where $\dot{M}$ does not differ too much from the CAK value. Gayley (2000) also finds that any process leading to mass-loss enhancement must reduce the terminal speed of the wind, conform with the findings of Poe et al. (1990).

In a recent series of papers, Feldmeier \& Shlosman (2000, 2002) and Feldmeier et al. (2002) have considered runaway of line-driven winds towards critical and overloaded solutions. Their physical model is a generalisation of the CAK model, in which they have assumed zero sound speed and $\alpha=0.5$ for all examples shown in these papers. A perturbation at a fixed height is fed into the wind such that the inner wind is lifted towards higher mass-loss rates. These solutions are accompanied by flow deceleration and kinks in the velocity at the point in the wind where the deceleration starts to occur. The increase in the mass loss these authors find amounts to only a few percent compared to the classical CAK mass loss.

It is important to have accurate predictions of the (maximum) mass-loss rates as a function of stellar parameters in order to derive reliable evolutionary models of massive stars. Indeed, the mass loss significantly affects the evolution of stars with initial masses above some $20 M_{\odot}$.
Maeder \& Meynet (2000a,b) have recently reviewed the effects of the interplay between rotation and mass loss on stellar evolution of massive stars. They come to the conclusion that the effects of rotation on the mass-loss rates remain moderate for stars sufficiently far from the Eddington limit. On the other hand, when the rotation-corrected Eddington factor $\Gamma$ is considerable, (with $\Gamma=g_{\mathrm{e}} / g_{\text {grav }}$ is the ratio between the radiative acceleration due to electron scattering and the accelaration of gravity corrected for centrifugal acceleration), even a moderate rotation rate can lead to extremely high mass loss. We will briefly discuss the effect of rotation on the maximum mass-loss rate in this paper. A subsequent paper (Aerts et al., in preparation) will be devoted completely to the mass loss of rotating hot stars based on our formalism but with the inclusion of gravity darkening. In that future paper we also provide a comparison to the predictions by Maeder \& Meynet (2000b) and Langer (1998).

This paper is organised as follows. In Sect. 2 we give a description of the different physical ingredients of our model. We derive the momentum equation for a CAK-type radiatively driven wind with a temperature law of the form $T(r) \sim r^{-x}$. We explicitly include the effect of photon tiring ${ }^{1}$. We also formulate the boundary conditions we adopted to solve the momentum equation. Subsequently, in Sect. 3, we illustrate our method by applying it to the stellar parameters of an O5IV star. In Sect. 4 we study the influence of different physical effects on the maximum mass-loss rate: photon tiring, the finite disk correction, the position of the sonic point and rotation. With that purpose, we apply our formalism to an O-type main-sequence star and a B-type supergiant. In Sect. 5 we provide maximum mass-loss rates for a grid of stellar parameters. Finally, we end this work with some concluding remarks.

\section{Description of the physical model}

The theory of radiation-driven winds was reviewed extensively before by many authors. We refer to Chap. 8 of the book by Lamers \& Cassinelli (1999) and to the review paper by Kudritzki \& Puls (2000), and references therein, for a basic extensive overview and limit ourselves here by giving the main concepts needed to understand our formalism. A concise but very clear short description of basic CAK theory can be found in e.g. Owocki \& Puls (2002, Sect. 2.3).

\subsection{The adopted line-driven wind formalism in the equatorial plane of a rotating star}

The basic expression for the line acceleration of a spherically symmetric stationary stellar wind is:

$g_{\mathrm{L}}=\frac{\sigma_{\mathrm{e}} L_{\star}}{4 \pi r^{2}}\left(k t^{-\alpha}\right) D_{\mathrm{fd}}$,

\footnotetext{
1 Photons that are multiply scattered in a wind lose energy as they transfer their momentum to the gas. This is called photon tiring, as it implies that the force produced by radiation pressure decreases for high mass-loss rates and may no longer be able to lift the wind out of the potential well of the star.
} 
where the first right-hand factor is the radiative acceleration by electron scattering for radiation from a point source, with $\sigma_{\mathrm{e}}$ being the electron scattering opacity per gram, the second factor is the force multiplier and the third takes into account the non-radial direction of the radiation. The force multiplier describes the radiative acceleration in terms of that by electron scattering where the scaling factor $k$ depends on the number of spectral lines and $t$ is a reference optical depth

$t=\sigma_{\mathrm{e}}^{\mathrm{ref}} v_{\mathrm{th}} \rho \frac{\mathrm{d} r}{\mathrm{~d} v}$,

with $\sigma_{\mathrm{e}}^{\text {ref }}$ a reference value of $0.325 \mathrm{~cm}^{2} \mathrm{~g}^{-1}$ (see e.g. Abbott 1982). The finite disk correction factor is given by:

$D_{\mathrm{fd}} \equiv \frac{(1+\sigma)^{\alpha+1}-\left(1+\sigma \mu_{\star}^{2}\right)^{\alpha+1}}{\left(1-\mu_{\star}^{2}\right)(\alpha+1) \sigma(1+\sigma)^{\alpha}}$,

where

$\sigma \equiv \frac{\mathrm{d} \ln v}{\mathrm{~d} \ln r}-1$ and $\mu_{\star} \equiv 1-\left(\frac{R_{\star}}{r}\right)^{2}$

(Pauldrach et al. 1986; see also Lamers \& Cassinelli 1999, Chap. 8 for a derivation). This finite disk correction factor takes into account that the radiation has a significant non-radial component close to the star. It was included in a very clever way in the description of radiation-driven winds of hot stars by Kudritzki et al. (1989).

For the wind from a rotating star, the radial component of the equation of motion in the equatorial plane of a line-driven wind is written as follows:

$v \frac{\mathrm{d} v}{\mathrm{~d} r}+\frac{G M_{\star}}{r^{2}}+\frac{1}{\rho} \frac{\mathrm{d} p}{\mathrm{~d} r}-v_{\mathrm{eq}}^{2} \frac{R_{\star}^{2}}{r^{3}}-g_{\mathrm{rad}}=0$,

where $v_{\text {eq }}$ stands for the equatorial rotation velocity and $g_{\text {rad }}$ is the acceleration due to both continuum and line radiation. The fourth term is the centrifugal force in the equatorial plane in case of conservation of angular momentum. For higher stellar latitudes $\theta$ the centrifugal force is smaller by a factor $\cos ^{2} \theta$ and vanishes in the polar direction. For this study we only consider the mass flux in the equatorial plane. We neglect the deformation of the star due to rotation. This is justified as flattening is only important for stars rotating close to their critical rate (Pelupessy et al. 2000).

Besides this equation, we consider also the equation of mass continuity:

$\dot{M}_{\mathrm{eq}} \equiv 4 \pi R_{\star}^{2} F_{m}^{\mathrm{eq}}=4 \pi r^{2} \rho(r) v(r)$

where $F_{m}^{\mathrm{eq}}$ is the mass flux (in $\mathrm{g} \mathrm{cm}^{-2} \mathrm{~s}^{-1}$ ) from the equatorial zone. This equation is valid if the wind moves radially outward i.e. if streaming of the wind from higher latitudes to the equatorial plane or vice versa is neglected.

The equation of state for a perfect gas is:

$p(r)=\rho(r) a(r)^{2}$,

with $a(r)$ the sound speed. We assume a non-isothermal stellar wind with the following temperature dependence:

$T(r)=T_{\mathrm{eff}}\left(\frac{R_{\star}}{r}\right)^{x}$, where $R_{\star}$ and $T_{\text {eff }}$ stand for the stellar radius and effective temperature respectively. We assumed a temperature structure with $x=1$. The effect of this temperature structure will be discussed in Sect. 4.3. In most studies the wind is considered to be isothermal, but since we will have to search for numerical solutions of the momentum equation anyway, we prefer a more realistic temperature distribution.

Substitution of the adopted temperature law into the equation of state, and subsequently into the equation of motion by making use of Eq. (6), multiplication by $r^{2}$, and substitution of the expression for the line-driving (1) leads to the following version of the equation of motion:

$$
\begin{aligned}
& \left(1-\frac{a_{\star}^{2} R_{\star}}{r v^{2}}\right) r^{2} v \frac{\mathrm{d} v}{\mathrm{~d} r}-3 R_{\star} a_{\star}^{2}-v_{\mathrm{eq}}^{2} \frac{R_{\star}^{2}}{r} \\
& +G M_{\star}-\frac{\sigma_{\mathrm{e}} L(r)}{4 \pi c}\left[1+D_{\mathrm{fd}} \frac{\sigma_{\mathrm{e}}^{\mathrm{ref}}}{\sigma_{\mathrm{e}}} k\left(\frac{4 \pi}{\sigma_{\mathrm{e}}^{\mathrm{ref}} v_{\mathrm{th}} \dot{M}_{\mathrm{eq}}}\right)^{\alpha}\right. \\
& \left.\quad \times\left(r^{2} v \frac{\mathrm{d} v}{\mathrm{~d} r}\right)^{\alpha}\right]=0,
\end{aligned}
$$

with $\dot{M}_{\text {eq }}$ the mass loss and $L(r)$ the radiative luminosity at distance $r$. This value does not have to be constant, as will be shown below. In this equation, $a_{\star}$ denotes the sound speed at the stellar surface.

We point out that we have omitted the factor $\left(n_{\mathrm{e}} / W\right)^{\delta}$, with $n_{\mathrm{e}}$ and $W$ respectively the electron density and the dilution factor, in our description of the radiative acceleration by lines, Eq. (1). This factor is connected to the reduction of the line force due to the change in ionisation in the wind. Its inclusion will only lower the upper limit of the maximum mass loss, so it is justified to neglect this factor for our purpose, which we have done in order to simplify finding solutions of Eq. (9). In our model we also neglect the instability of the radiative line force, which is a good approximation if the goal is to study the large-scale wind behaviour (for a review of such wind instabilities, see Owocki 1994).

\subsection{The inclusion of photon tiring}

The concept of photon tiring was first put forward by Owocki \& Gayley (1997). The authors used this term to indicate that, for a star with a given luminosity, only a limited mass-loss rate can be accelerated before the energy expended in accelerating the outflow becomes a considerable fraction of the original stellar luminosity. Owocki \& Gayley took into account the effect of photon tiring by reducing the stellar luminosity according to the gained kinetic and potential energy of the flow at each distance in the wind. They performed some preliminary calculations of acceptable mass-loss rates for a specific form of the Eddington factor. This form was such that it allowed to determine the effect of photon tiring on the mass loss analytically, for illustrative purposes. In this paper, we include the effect of photon tiring with a more realistic description. 
In order to take into account the effect of photon tiring, we express the luminosity $L(r)$ in Eq. (9) as

$$
\begin{aligned}
L(r)= & L_{\star}-\frac{\dot{M}_{\mathrm{eq}}}{2}\left(v^{2}(r)-a_{\star}^{2}\right)-\frac{G M_{\star} \dot{M}_{\mathrm{eq}}}{R_{\star}}\left(1-\frac{R_{\star}}{r}\right) \\
& +\frac{5}{2} \dot{M}_{\mathrm{eq}} a_{\star}^{2}\left(1-\frac{R_{\star}}{r}\right),
\end{aligned}
$$

where $L_{\star}$ is the radiative luminosity from the photosphere. The right-hand side of this equation is obtained by expressing conservation of kinetic and potential energy, as well as enthalpy, from the stellar radius onwards throughout the wind for the temperature law adopted in (8).

Evaluation of Eq. (10) shows that the fractional change of the stellar luminosity always is very small for all stellar models considered in this paper, suggesting the effect of photon tiring to be negligible. We have determined explicitly the effect of photon-tiring by using Eq. (10) when integrating the momentum equation. For none of the mass-loss rates and velocity laws given in the paper foton tiring plays any role. We therefore omit further discussion of this effect.

\subsection{Solving the momentum equation as a function of mass loss}

The traditional approach to solve the momentum Eq. (9) is to search for the critical point of the equivalent of this equation for an isothermal wind, by imposing a singularity and a regularity condition, which both are a function of the mass loss $\dot{M}$. These conditions are constructed in such a way that a smooth transsonic solution is obtained, from which the mass loss $\dot{M}$ is determined for a self-regulating or perturbed wind. For the isothermal analogue of Eq. (9), the critical point is typically located around $1.1 R_{\star}$ (Kudritzki et al. 1989) and is not necessarily equal to the sonic point $R_{\mathrm{S}}$ (Castor et al. 1975; Lamers \& Cassinelli 1999, Chap. 8). From here on, we use the term classical mass-loss rates for such determination of $\dot{M}$.

In the current work, we take a different attitude. We are interested in solving the question: what is the maximum mass loss for which the momentum Eq. (9) still has an outflowing wind that escapes to infinity? In other words, we search for the maximum $\dot{M}_{\text {eq }}$, termed $\dot{M}_{\text {max }}^{\text {eq }}$, for which Eq. (9) still has a solution ánd for which this solution corresponds to an outflow velocity law at all distances. The velocity does not have to be monotonically increasing outwards, but it has to remain positive. We determine $\dot{M}_{\text {max }}^{\text {eq }}$ by solving Eq. (9) for increasing values of $\dot{M}_{\text {eq }}$ until the equation is no longer solvable because the line force has become too weak. Out of this pool of solutions, we select $\dot{M}_{\text {max }}^{\text {eq }}$ by picking out the largest one for which the condition of a positive wind velocity at large distance of the stellar surface still holds.

As boundary condition, we impose the velocity gradient at the sonic point and we search for solutions from the sonic point up to $r \rightarrow+\infty$. We assume the sonic point to be located somewhere in the interval $R_{\mathrm{S}} \in[1.01,1.1] R_{\star}$. The velocity gradient in $R_{\mathrm{S}}$ is determined numerically by solving the equation:

$$
\begin{gathered}
\left(1-\frac{a_{\star}^{2} R_{\star}}{R_{\mathrm{s}} a\left(R_{\mathrm{s}}\right)^{2}}\right) R_{\mathrm{s}}^{2} a\left(R_{\mathrm{s}}\right)\left(\frac{\mathrm{d} v}{\mathrm{~d} r}\right)_{r=R_{\mathrm{s}}}-3 R_{\star} a_{\star}^{2}-v_{\mathrm{eq}}^{2} \frac{R_{\star}^{2}}{R_{\mathrm{s}}} \\
+G M_{\star}-\frac{\sigma_{\mathrm{e}} L_{\star}}{4 \pi c}\left[1+\frac{\sigma_{\mathrm{e}}^{\mathrm{ref}}}{\sigma_{\mathrm{e}}} k\left(\frac{4 \pi}{\sigma_{\mathrm{e}}^{\mathrm{ref}} v_{\mathrm{th}} \dot{M}_{\mathrm{eq}}}\right)^{\alpha}\right. \\
\left.\quad \times\left(D_{\mathrm{fd}}\right)_{r=R_{\mathrm{s}}}\left(R_{\mathrm{s}}^{2} a\left(R_{\mathrm{s}}\right)\left(\frac{\mathrm{d} v}{\mathrm{~d} r}\right)_{r=R_{\mathrm{s}}}\right)^{\alpha}\right]=0,
\end{gathered}
$$

which is the momentum equation for a fixed location of the sonic point. Since all factors are known for any adopted value of $R_{\mathrm{s}}$, the value of $(\mathrm{d} v / \mathrm{d} r)_{R_{\mathrm{s}}}$ can be found.

Imposing the position of the sonic point allows one to find solutions of the momentum equation without encountering delicate mathematical complexities compared to making use of the singularity and regularity condition. It also admits inclusion of additional effects, such as photon tiring and non-isothermal decelerated flows. The objective of our study is to investigate how high the mass-loss rate can become in solving the momentum equation under these conditions.

\section{Illustration of the method}

In the following we solve the equation of motion (9) as described in Sect. 2.2 and determine $\dot{M}_{\max }^{\mathrm{eq}}$ for one particular stellar model assuming that the sonic point is situated at $1.1 R_{\star}$. This choice of $R_{\mathrm{s}}$ hardly affects the resulting mass-loss rate, because we only solve for the supersonic part of the wind. We will illustrate this further in the paper.

We consider the stellar parameters corresponding to an O5IV star - see Table 1. Accurate values of the CAK line parameters were determined from NLTE calculations by Pauldrach et al. (1986). For the effective temperature of an O5IV star they find $k=0.124, \alpha=0.64$. Further, we have used $\sigma_{\mathrm{e}}=0.34 \mathrm{~cm}^{2} \mathrm{~g}^{-1}$ for the O5IV star.

We solve the momentum equation numerically by means of a code written in Mathematica ${ }^{2}$ with the boundary condition of $(\mathrm{d} v / \mathrm{d} r)_{R_{\mathrm{s}}}$ of Eq. (11) described above. We perform the integrations of the equation for increasing values of $\dot{M}_{\text {eq }}$ in steps of $10^{-7} M_{\odot} \mathrm{yr}^{-1}$ and search for $\dot{M}_{\max }^{\mathrm{eq}}$ as described in the previous section.

In a first example we have considered the O 5 IV star to have an equatorial rotation velocity of $v_{\text {eq }}=300 \mathrm{~km} \mathrm{~s}^{-1}$, which is about half the critical velocity. We have determined solutions to the momentum equation for increasing values of the equatorial mass loss. The courses of the accompanying wind velocities $v(r)$ in the equatorial plane are graphically depicted in Fig. 1. We find a maximum equatorial mass-loss rate of $2.7 \times 10^{-6} M_{\odot} \mathrm{yr}^{-1}$ for this case. Increasing the mass-loss rate does no longer allow to find solutions of the momentum equation. The terminal wind velocity amounts to some $450 \mathrm{~km} \mathrm{~s}^{-1}$ in this example. For comparison, we mention that the classical CAK solution for this stellar configuration is

\footnotetext{
${ }^{2}$ Code available upon request.
} 


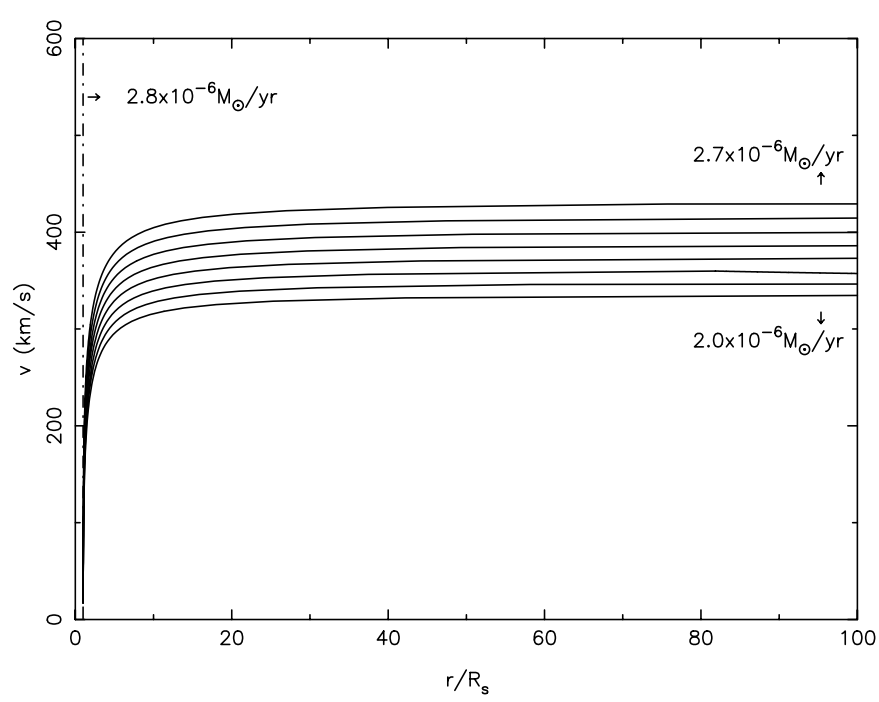

Fig. 1. Different solutions of the momentum equation for the stellar parameters of an O5IV star (see Table 1) rotating at $v_{\text {eq }}=300 \mathrm{~km} \mathrm{~s}^{-1}$. The solutions for increasing mass-loss rates are indicated as full lines. The dashed-dot line indicates graphically that the momentum equation is no longer solvable for the indicated and higher mass-loss rates.

$\dot{M}_{\mathrm{CAK}}=3.7 \times 10^{-6} M_{\odot} \mathrm{yr}^{-1}$ and $v_{\infty}=1257 \mathrm{~km} \mathrm{~s}^{-1}$ (this CAK velocity law is not shown in Fig. 1 as it would hinder the visibility of the different non-CAK curves). The fact that the CAK mass loss is higher than the maximum mass loss is entirely due to the neglect of the finite disk correction in the classical CAK model. Including the finite disk correction gives a modified CAK mass loss of $\dot{M}_{\mathrm{mCAK}}=2.3 \times 10^{-6} M_{\odot} \mathrm{yr}^{-1}$ and $v_{\infty}=2325 \mathrm{~km} \mathrm{~s}^{-1}$. The velocity law corresponding to the maximum mass-loss rate has a much lower terminal speed than both these CAK solutions, as our formalism is such that all effort is done to drive as high a mass loss as possible, at the expense of a high velocity (see also Fig. 4 in Poe et al. 1990).

For all rotation velocities between 0 and $450 \mathrm{~km} \mathrm{~s}^{-1}$, i.e. between $0 \%$ and $80 \%$ of the critical velocity, we find similar types of results as the one shown in Fig. 1.

In a subsequent example we adopted an equatorial rotation velocity of $v_{\text {eq }}=500 \mathrm{~km} \mathrm{~s}^{-1}$, which is $90 \%$ of the critical velocity. The variation of the radial velocity $v(r)$ in the equatorial plane is shown in Fig. 2. In this case of near-critical rotation, we find two types of physically relevant solutions:

1. solutions for which the line force is able to drive the mass towards an infinite distance from the star (full lines);

2. solutions for which the line force is only able to drive the mass to a limited distance (dashed lines).

The dashed-dot line indicates again the treshold in mass loss for which the momentum equation is no longer solvable at any distance from the star.

In this example, solutions of type 1 are found for mass-loss rates up to $3.6 \times 10^{-6} M_{\odot} \mathrm{yr}^{-1}$. After having found the highest mass-loss rate accompanied by an overall increasing velocity law, we can still find solutions with higher mass-loss rates in such a way that the stellar wind material is lifted only to a certain limited distance from the star. Such solutions of type 2 are also valid, provided that the wind material has already accumulated a sufficient amount of energy at that distance to escape from the star. This requires that the wind velocity at the point where the radiative acceleration is no longer effective is higher than the local escape velocity, or rather that the sum of the kinetic and potential energy and the enthalpy per unit mass is positive.

Increasing the mass loss to $3.8 \times 10^{-6} M_{\odot} \mathrm{yr}^{-1}$ implies that the line driving is only able to lift the wind material to a distance of some $12 R_{\mathrm{s}}$, after which it can no longer gain momentum. From that point onwards the velocity starts to decrease according to the transfer of kinetic energy into potential energy and enthalpy (see Eq. (10) above). For a mass loss up to $4.0 \times 10^{-6} M_{\odot} \mathrm{yr}^{-1}$ the wind material has sufficient energy to escape from the star, while increasing the mass loss to $4.1 \times 10^{-6} M_{\odot} \mathrm{yr}^{-1}$ results in wind material falling back to the star. In this example, we thus obtain $\dot{M}_{\max }^{\mathrm{eq}}=4.0 \times$ $10^{-6} M_{\odot} \mathrm{yr}^{-1}$ and the maximum wind velocity $v_{\max }$ amounts to some $400 \mathrm{~km} \mathrm{~s}^{-1}$. This maximum velocity is no longer the terminal velocity, which will approach zero at the maximum mass-loss rate.

A velocity kink in the wind speed similar the one we find for the models of the O5IV star near critical rotation $(>80 \%)$ was recently also found by Porter \& Skouza (1999) and Krtička \& Kubát (2000), who studied velocity deceleration by considering respectively ionization cutoff and decoupling of the gas and the radiation field in low-density radiately-driven winds. However, these authors did not connect these kinked velocity laws to a detailed study of the mass loss. The latter was done in the already cited recent papers by Feldmeier \& Shlosman (2000, 2002), resulting in a maximum of $9 \%$ increase of the classical mass-loss rate. This is comptabile with the difference we find between the maximum equatorial mass loss as we have determined it and the modified CAK mass loss for our first example.

\section{The influence of the physical effects on the maximum mass-loss rate}

In order to investigate the effects of the different physical ingredients of our model, we have determined the maximum massloss rates for a typical O5IV and B5II star of which the relevant stellar parameters are listed in Table 1. We have done this for different equatorial rotation velocities, ranging from $0 \mathrm{~km} \mathrm{~s}^{-1}$ up to a value close to the limiting rotation velocity. The latter is defined in this paper as the highest possible equatorial rotation velocity for which the velocity gradient in the sonic point derived from Eq. (11) is still positive, i.e. it is the critical rotation velocity at the sonic point. This limiting velocity is determined numerically; we find $v_{\mathrm{lim}} \sim 525 \pm 5 \mathrm{~km} \mathrm{~s}^{-1}$ for the O5IV star and $\sim 430 \pm 5 \mathrm{~km} \mathrm{~s}^{-1}$ for the B5II star. We note that, classically, the critical velocity at the stellar photosphere is usually defined as

$v_{\text {crit }}^{2} \equiv \frac{G M_{\star}}{R_{\star}}\left(1-\Gamma_{\mathrm{e}}\right)$

where $\Gamma_{\mathrm{e}}=\sigma_{\mathrm{e}} L_{\star} / 4 \pi G M_{\star} c$ stands for the Eddington ratio corresponding to the electron scattering opacity $\sigma_{\mathrm{e}}$. This definition 

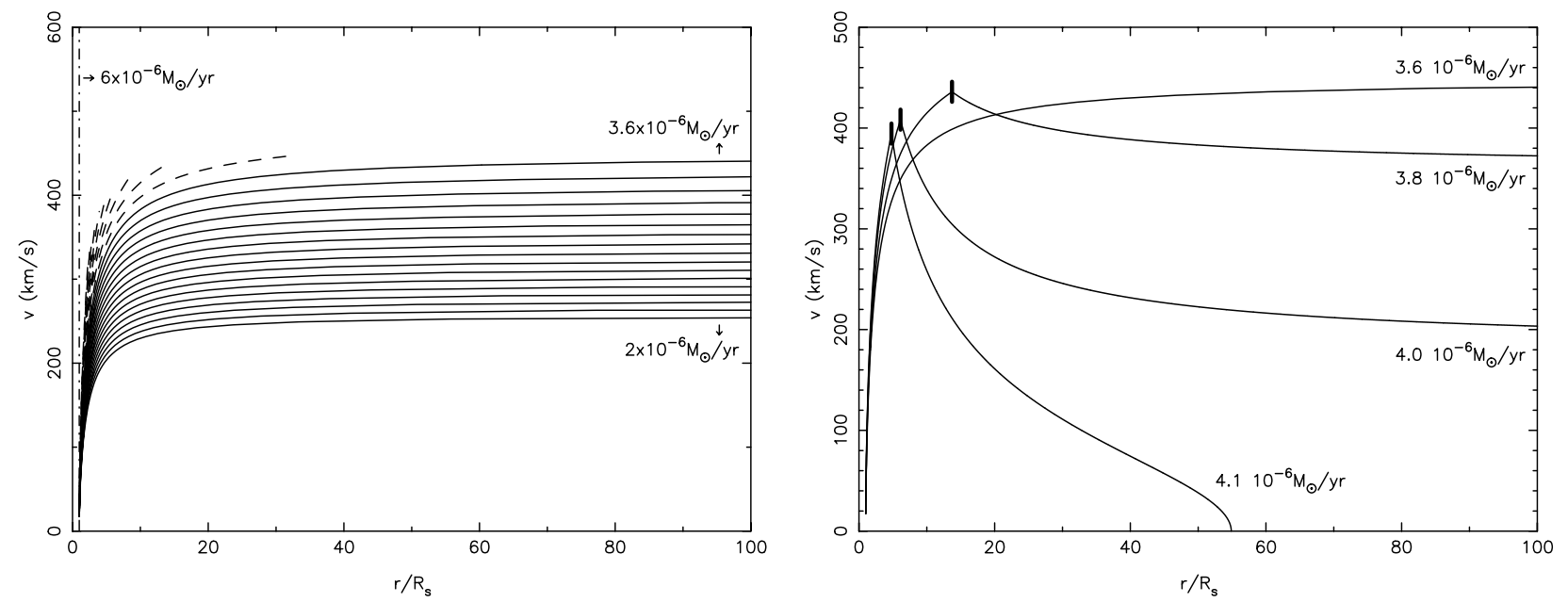

Fig. 2. Different solutions of the momentum equation for the stellar parameters of an O5IV star (see Table 1 ) rotating at $v_{\mathrm{eq}}=500 \mathrm{~km} \mathrm{~s}{ }^{-1}$. Left panel: solutions with increasing velocities towards infinity are indicated as full lines; the dashed lines are solutions for which the line force is exhausted at a certain distance. The dashed-dot line indicates graphically that the momentum equation is no longer solvable for the indicated and higher mass-loss rates. Right: selected solutions giving the transition from infinitely increasing to decreasing velocity laws. The thick vertical line segment indicates the position at which line driving is no longer effective.
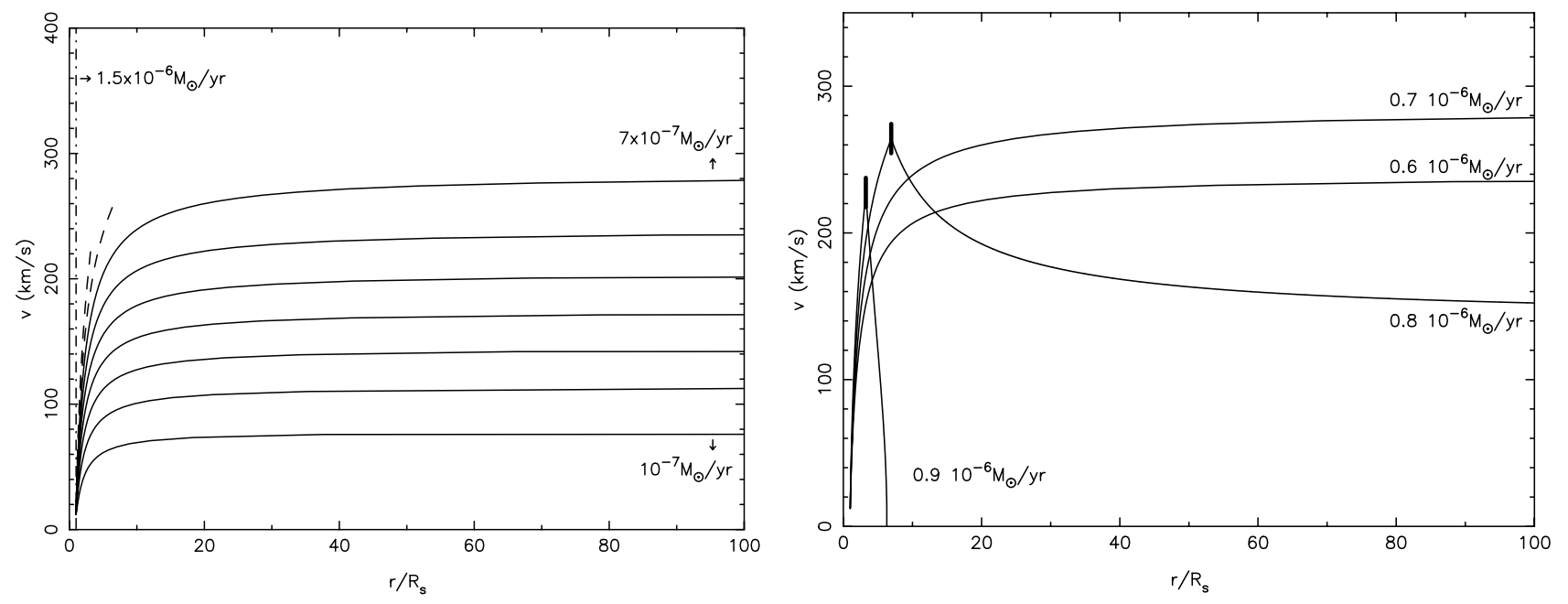

Fig. 3. Same as Fig. 2, but for a B5II star rotating at $v_{\mathrm{eq}}=400 \mathrm{~km} \mathrm{~s}^{-1}$.

Table 1. Stellar parameters of the O5IV and an B5II star for which we determined the maximum mass-loss rates as a function of equatorial rotation velocity. $\alpha$ and $k$ are taken from Pauldrach et al. (1986). The classical CAK mass loss and CAK terminal wind velocity are also listed. $\sigma_{\mathrm{e}}$ is expressed in $\mathrm{cm}^{2} \mathrm{~g}^{-1}, \dot{M}_{\mathrm{CAK}}$ in $M_{\odot} \mathrm{yr}^{-1}$ and $v_{\infty}$ in $\mathrm{km} \mathrm{s}^{-1}$.

\begin{tabular}{|c|c|c|c|c|c|c|c|c|c|c|c|c|c|}
\hline star & $\log T_{\text {eff }}$ & $\log L_{\star} / L_{\odot}$ & $M_{\star}\left(M_{\odot}\right)$ & $R_{\star}\left(R_{\odot}\right)$ & $v_{\text {crit }}$ & $R_{\mathrm{S}}\left(R_{\odot}\right)$ & $v_{\lim }$ & $\overline{\Gamma_{\mathrm{e}}}$ & $\alpha$ & $k$ & $\sigma_{\mathrm{e}}$ & $\dot{M}_{\mathrm{CAK}}$ & $v_{\infty}$ \\
\hline O5IV & 4.60 & 5.7 & 40 & 18 & 535 & 19.8 & 525 & 0.326 & 0.640 & 0.124 & 0.34 & $3.7210^{-6}$ & 1257 \\
\hline B5II & 4.30 & 5.0 & 30 & 30 & 435 & 33.0 & 430 & 0.016 & 0.565 & 0.320 & 0.31 & $0.6510^{-6}$ & 1074 \\
\hline
\end{tabular}

applies when the brightness of the star over its surface is uniform, as we are assuming here. We compared $v_{\text {crit }}$ and $v_{\text {lim }}$ for all the cases calculated in this work and it turns out that their values are very similar, differing less than 5\% from each other. We therefore always list any result with respect to $v_{\text {lim }}$, as this quantity is consistently connected to our calculations.

As in the illustrative example explained in the previous section we determine the solution of the momentum Eq. (9) for increasing values of $\dot{M}_{\mathrm{eq}}$, in steps of $10^{-7} M_{\odot} \mathrm{yr}^{-1}$. A similar plot as Fig. 2, but now for the B5II star rotating with $400 \mathrm{~km} \mathrm{~s}^{-1}$, which is $93 \%$ of the critical velocity, is shown in Fig. 3. From the right panel of Fig. 3 we see that the highest possible mass loss associated with an increasing velocity law amounts to $8 \times 10^{-7} M_{\odot} \mathrm{yr}^{-1}$ for the B 5 supergiant. The conclusion from all the different testcases is that increasing velocity laws are found for rotational velocities below $80 \%$ of the critical velocity. For higher rotation velocities the situation is always such that, once we have derived the highest mass loss with an overall increasing velocity law, only slightly higher mass-loss rates can 
be driven having a kinked velocity law with a still outflowing wind.

The velocity laws that are found by integrating the momentum equation have low terminal velocities. Models with massloss rates very close to the maximum value, i.e. models where radiative acceleration is no longer able to provide acceleration, have an outward decreasing wind velocity at high distance as illustrated in the right panels of Figs. 2 and 3. All models with mass-loss rates not too close to the maximum value show an outward increasing velocity law which can be approximated reasonably well with a $\beta$-law of the type $v(r) \simeq v_{\infty}\left(1-R_{\mathrm{S}} / r\right)^{\beta}$. This is shown in Fig. 4 for the O5IV star rotating at $400 \mathrm{~km} \mathrm{~s}^{-1}$, which is $72 \%$ of the critical velocity, for which we still found an increasing velocity towards infinity, i.e. for which the line driving is effective all the way through the wind. On the same plot we show different $\beta$-type velocity laws with the same terminal velocity. It can be seen that the derived velocity law can be approximated by a $\beta$-law with $\beta \simeq 0.4$ or 0.5 close to the star at $r<10 R_{\mathrm{s}}$. However, for larger distances the velocity law approaches that of a higher value of $\beta \simeq 1$. The velocity laws of classical line-driven CAK-winds but with the finite disk effect taken into account have a velocity law that can be approximated with a $\beta \simeq 0.8$ law (Kudritzki et al. 1989; Lamers \& Cassinelli 1999).

The observed terminal velocities of OB stars (see Prinja et al. 1990; Lamers et al. 1995; Puls et al. 1996) are about 4-7 times higher than the $v_{\mathrm{lim}}$-values we find. This implies that most OB stars for which $v_{\infty}$ can be determined are not in the very outermost regime of maximum mass loss.

Conservation of energy determines the velocity behaviour at large distance for the highest possible mass-loss rates. This is illustrated in Fig. 5, which shows the predicted velocity laws of the O5IV star (left) and the B5II star (right).

\subsection{Effect of finite disk correction}

One important question is in how far the finite disk correction factor $D_{\mathrm{fd}}$ given by Exp. (3) influences the maximum mass-loss rates. As a check of the calculations, this factor was in each case determined a posteriori, i.e. after having solved the momentum equation. We found that $D_{\mathrm{fd}}$ is smaller than 1 at small distances and larger than 1 at large distances from the star. The values of $D_{\mathrm{fd}}<1$ are due to the non-radial direction of the radiation close to the star, whereas $D_{\mathrm{fd}}>1$ is due to the fact that the line optical depth is smaller in the non-radial direction than in the radial direction (e.g. Kudritzki et al. 1989; Lamers \& Cassinelli 1999).

In order to assess the influence of $D_{\mathrm{fd}}$, we have determined $\dot{M}_{\max }^{\text {eq }}$ from Eq. (9) in which we have set $D_{\mathrm{fd}}=1$ and compared this to the solutions taking into account the true behaviour of the finite disk correction factor. The results of such a comparison are graphically illustrated in Fig. 5 (dashed line: without $D_{\mathrm{fd}}$; full line: with $D_{\mathrm{fd}}$ ). We find that the inclusion of finite disk correction leads in general to slightly higher mass-loss rates, contrary to the situation in self-regulating CAK winds. The explanation for this is that $D_{\mathrm{fd}}<1$ in only a very limited regime very close to the star (in a self-regulated wind this is

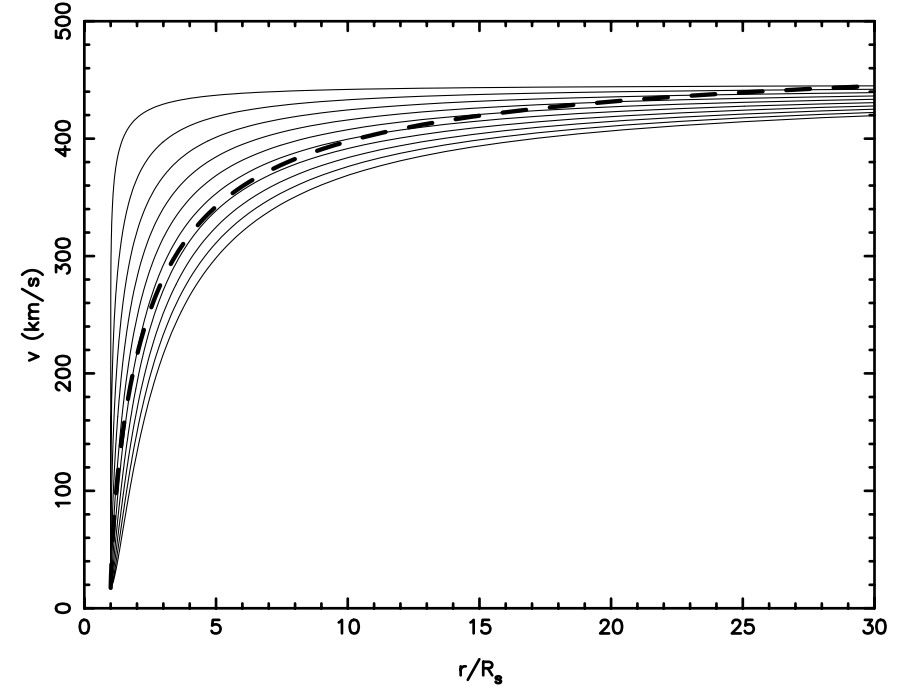

Fig. 4. A comparison between a velocity law found from Eq. (9) (dashed line) and different $\beta$ velocity laws (full lines). The latter are drawn for $\beta=0.1$ (upper curve) $\rightarrow 1.9$ (lower curve) in steps of 0.2 .

where the value of the mass loss is determined), while the maximum mass loss is mainly dependent on the velocity behaviour further out in the wind, at a few stellar radii, where $D_{\mathrm{fd}}>1$. Hence, the finite disk correction helps in a slightly positive way in driving the wind in the region $R>2 R_{\star}$. Although increases in the maximum mass-loss rates occur by including $D_{\mathrm{fd}}$, they always remain small, e.g. below $10^{-7} M_{\odot} \mathrm{yr}^{-1}$ in the two examples mentioned in Table 1.

\subsection{Effect of the position of the sonic point}

Up to now the sonic point was always assumed to be positioned at $1.1 R_{\star}$. Subsequently, we have investigated the effect of changing the position of the sonic point by varying its position between 1.01 and $1.1 R_{\star}$. We illustrate our findings for the velocity law corresponding to the highest mass loss for the O 5 II star in Fig. 6. It can be seen from this plot that our results are quite robust against the choice of the position of the sonic point within reasonable limits. The further out the sonic point, the more difficult it is to drive a high mass loss as the velocity gradient is less steep. Changing the sonic point within the indicated limits does not change the velocity law for the B-supergiant. With the goal to find the maximum mass loss we therefore assume the sonic point to be located at $1.01 R_{\star}$ in all the calculations done in Sect. 5 .

The temperature structure of winds from hot stars has been discussed by e.g. de Koter et al. (1993), who found that the temperature does not decrease very steeply very far out in the wind (see their Fig. 1). Presumably, the precise temperature law does not matter too much for our study, as the effect of the gass pressure is very limited compared to the one of the radiation pressure. In order to assess this statement more quantitatively, we have also performed some calculations in which we have taken $x=0.5$ instead of $x=1$ in the temperature law (8). We have adjusted Eqs. (9), (11), and (12) accordingly and have determined again $\dot{M}_{\max }^{\mathrm{eq}}$ and $v_{\max }$ for this less steep temperature 

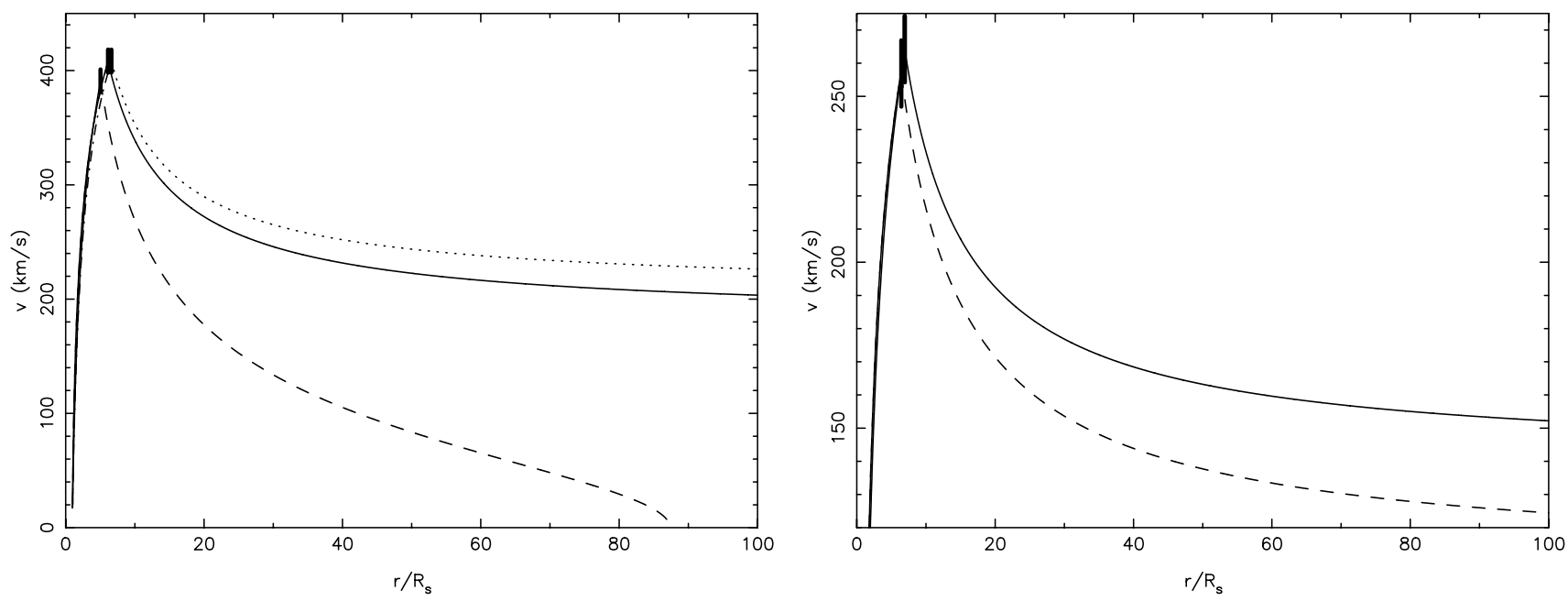

Fig. 5. Left panel: the velocity law for the O5IV star having $v_{\mathrm{eq}}=500 \mathrm{~km} \mathrm{~s}^{-1}$ and $\dot{M}_{\mathrm{eq}}=4.0 \times 10^{-6} M_{\odot} \mathrm{yr}^{-1}$. Full line: inclusion of finite disk correction. Dashed line: neglect of finite disk correction. Dotted line: same as dashed line, but for $\dot{M}_{\mathrm{eq}}=3.9 \times 10^{-6} M_{\odot} \mathrm{yr}^{-1}$. Right panel: same as left panel but for the velocity law of the B5II star having $v_{\text {eq }}=400 \mathrm{~km} \mathrm{~s}^{-1}$ and $\dot{M}_{\text {eq }}=0.8 \times 10^{-6} M_{\odot} \mathrm{yr}^{-1}$.

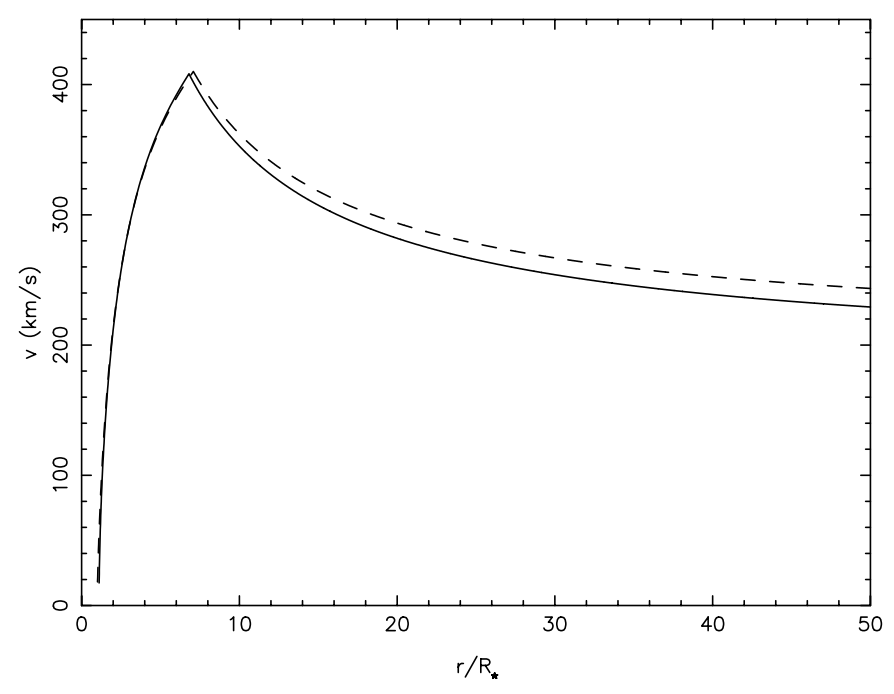

Fig. 6. The velocity law for the O5IV star having $v_{\mathrm{eq}}=500 \mathrm{~km} \mathrm{~s}^{-1}$ and $\dot{M}_{\text {eq }}=4 \times 10^{-6} M_{\odot} \mathrm{yr}^{-1}$ with the inclusion of finite disk correction. The sonic point was fixed at $1.1 R_{\star}$ (full line) and at $1.01 R_{\star}$ (dashed line).

law. This indeed does not change the values of these two quantities so that our proposed formalism is sufficiently accurate as a general description of maximum mass loss.

\subsection{Effect of rotation}

An important effect that also influences the mass loss of the star is its rotational velocity. Pioneering studies of the effect of rotation on radiatively-driven wind models were done by Poe \& Friend (1986) and Friend \& Abbott (1986). Poe \& Friend (1986) found that the mass-loss rates depend very strongly on the equatorial rotation velocity, with typically a factor 5 difference in mass loss between slow and near-critical rotation, for magnetic Be star models. Friend \& Abbott (1986) showed convincingly that the terminal wind speed decreases due to the inclusion of the centrifugal force, while the mass loss increases with at most a factor 5, in agreement with Poe \& Friend's result. The question is what kind of factor between the maximum mass-loss rates of non-rotating and of near-critical rotation we find from our formalism.

In the present paper we discuss the effect of rotation on the maximum equatorial mass flux only. We have investigated the behaviour of the maximum mass loss derived with the method explained above as a function of the equatorial rotation velocity. We stress that we do not take into account gravitational darkening of the fast rotating star, which reduces the radiative flux at the equator compared to the pole (Von Zeipel effect), nor the effect of the increased equatorial radius by rotational flattening. This means that our calculated maximum equatorial mass flux should be compared with that of a non-rotating star with the same gravity, radius and $T_{\mathrm{eff}}$ as in the rotating star at the equator. The effect of rotation on the maximum overall, i.e. surface integrated, mass loss, with gravity darkening taken into account, will be studied in a separate paper (Aerts et al., in preparation).

The results for $\dot{M}_{\max }^{\mathrm{eq}}$ with the inclusion of foton tiring and finite disk correction are listed in Table 2 as a function of equatorial rotation velocity for the case where the sonic point is situated at $1.1 R_{\star}$. We find that the maximum mass-loss rates for the $\mathrm{O} 5$ main-sequence star range from $2.2-4.0 \times 10^{-6} M_{\odot} \mathrm{yr}^{-1}$ and for the B 5 supergiant from $0.40-0.80 \times 10^{-6} M_{\odot} \mathrm{yr}^{-1}$. So rotation increases the maximum equatorial mass flux by only about a factor 2, even for near-critical rotation. Including the effect of the increase in the equatorial radius of a rapidly rotating star by at most a factor 1.5 implies that the maximum equatorial mass-loss rate of a rapidly rotating star can be about a factor 3 higher than the maximum equatorial mass loss rate of a non-rotating star with similar equatorial radiative flux and gravity, within the same narrow band of stellar latitude. Our results are compatible with the specific examples for Be stars treated by Owocki et al. (1994).

Since the maximum mass-loss rate of a non-rotating star is not much higher than the value predicted by classical 
Table 2. Maximum mass-loss rates (expressed in $10^{-6} M_{\odot} \mathrm{yr}^{-1}$ ) for the stars with stellar parameters listed in Table 1 as a function of equatorial rotation velocity $v_{\mathrm{eq}}$ for $R_{\mathrm{s}}=1.1 R_{\star}$. All $v_{\mathrm{max}}$-values are very similar. The listed velocities are all expressed in $\mathrm{km} \mathrm{s}^{-1}$.

\begin{tabular}{|c|c|c|c|c|c|c|c|c|c|}
\hline \multicolumn{5}{|c|}{ O5IV, $v_{\max } \simeq 450, v_{\text {lim }}=555, v_{\text {crit }}=535$} & \multicolumn{5}{|c|}{ B5II, $v_{\max } \simeq 280, v_{\text {lim }}=430, v_{\text {crit }}=435$} \\
\hline $\begin{array}{r}v_{\text {eq }} \\
\left(\mathrm{km} \mathrm{s}^{-1}\right) \\
\end{array}$ & $v_{\text {eq }} / v_{\text {lim }}$ & $\begin{array}{c}\dot{M}_{\max }^{\mathrm{eq}} \\
\left(10^{-6} M_{\odot} \mathrm{yr}^{-1}\right) \\
\end{array}$ & $\begin{array}{c}\dot{M}_{\mathrm{max}}^{\mathrm{eq}}\left(v_{\mathrm{eq}}\right) / \\
\dot{M}_{\mathrm{max}}^{\mathrm{eq}}(0) \\
\end{array}$ & $\eta_{\mathrm{eq}}$ & $\begin{array}{r}v_{\mathrm{eq}} \\
\left(\mathrm{km} \mathrm{s}^{-1}\right) \\
\end{array}$ & $v_{\mathrm{eq}} / v_{\mathrm{lim}}$ & $\begin{array}{c}\dot{M}_{\max }^{\mathrm{eq}} \\
\left(10^{-6} M_{\odot} \mathrm{yr}^{-1}\right) \\
\end{array}$ & $\begin{array}{c}\dot{M}_{\mathrm{max}}^{\mathrm{eq}}\left(v_{\mathrm{eq}}\right) / \\
\dot{M}_{\max }^{\mathrm{eq}}(0) \\
\end{array}$ & $\eta_{\mathrm{eq}}$ \\
\hline 0 & 0.00 & 2.20 & 1.00 & 0.10 & 0 & 0.00 & 0.40 & 1.00 & 0.05 \\
\hline 100 & 0.18 & 2.27 & 1.03 & 0.10 & 100 & 0.23 & 0.44 & 1.10 & 0.06 \\
\hline 150 & 0.27 & 2.34 & 1.06 & 0.10 & 150 & 0.35 & 0.48 & 1.20 & 0.07 \\
\hline 200 & 0.36 & 2.40 & 1.09 & 0.10 & 200 & 0.47 & 0.54 & 1.35 & 0.07 \\
\hline 250 & 0.45 & 2.56 & 1.16 & 0.11 & 250 & 0.58 & 0.60 & 1.50 & 0.08 \\
\hline 300 & 0.54 & 2.68 & 1.22 & 0.12 & 300 & 0.70 & 0.70 & 1.75 & 0.09 \\
\hline 350 & 0.63 & 3.00 & 1.36 & 0.13 & 350 & 0.81 & 0.78 & 1.95 & 0.11 \\
\hline 400 & 0.72 & 3.40 & 1.55 & 0.15 & 400 & 0.93 & 0.80 & 2.00 & 0.11 \\
\hline 450 & 0.81 & 3.90 & 1.77 & 0.17 & 420 & 0.98 & 0.80 & 2.00 & 0.11 \\
\hline 500 & 0.90 & 4.00 & 1.82 & 0.17 & - & - & - & - & - \\
\hline 550 & 0.99 & 4.00 & 1.82 & 0.17 & - & - & - & 一 & - \\
\hline
\end{tabular}

radiation driven wind models, within about a factor two (see below), we conclude that it is not possible to drive significantly higher mass-loss rates by line radiation pressure than predicted with the CAK theory. This is essentially also in accord with the findings of Gayley (2000) who has considered a much simpler treatment than we have done. We point out that Maeder \& Meynet (2000b) do find very high classical mass-loss rates for rotating stars with a high Eddington factor of $\Gamma_{\mathrm{e}}>0.64$. We did not consider this regime in the present work.

The predicted maximum wind velocity turns out to be independent of $v_{\text {eq }}$. One can understand this, as with our formalism, we concentrate entirely on increasing the mass loss as much as possible, irrespective of the consequences for the velocity law, provided that the wind material can still escape from the star. We have also calculated the equatorial momentum transfer efficiency $\eta_{\text {eq }}$, which we define in this paper as

$\eta_{\mathrm{eq}} \equiv \frac{\dot{M}_{\max }^{\mathrm{eq}} v_{\max }}{L_{\star} / c}$

This expression differs from that normally used $\eta=$ $\dot{M} v_{\infty} /\left(L_{\star} / c\right)$ because in our models $v_{\max }$ differs strongly from $v_{\infty}$, which is zero in the maximum mass loss models. The values are listed in Table 2 . As the maximum wind velocity is independent of $v_{\text {eq }}, \eta_{\text {eq }}$ 's dependence on $v_{\text {eq }}$ is the same as the one of $\dot{M}_{\max }^{\text {eq }}$. So $\eta_{\text {eq }}$ increases by about a factor two or three from non-rotating to fast rotating stars with the same equatorial values of $T_{\text {eff }}$ and effective gravity. The values of $\eta_{\text {eq }}$ of our models are smaller than those of the classical line-driven wind models, because $\dot{M}_{\text {max }}^{\text {eq }}$ is not much higher than $\dot{M}_{\mathrm{CAK}}$ whereas $v_{\max }$ is about a factor two smaller than $v_{\infty}^{\mathrm{CAK}}$.

A most important conclusion from Table 2 is that we find a finite maximum mass-loss rate at the limiting velocity from our formalism. The dependence of the mass-loss rate on the rotation of the star is the subject of an intensive, still ongoing debate (see, e.g. Maeder \& Meynet 2000b). We hence devote a special paper to this topic, in which we study the dependence of $\dot{M}_{\max }^{\text {eq }}$ on $v_{\text {eq }}$ in much more detail, including also gravity darkening, for all the stellar models considered in the next section (Aerts et al., in preparation) and compare our results with Maeder \& Meynet's.

\section{Maximum mass-loss rates as a function of stellar parameters}

In order to provide more systematically determined maximum mass-loss rates, we determined $\dot{M}_{\max }^{\mathrm{eq}}$ in the same way as the ones given in the previous section for a grid of nine stellar models typical for massive stars in the upper part of the HR diagram. The characteristics of these models are given in Table 3. The corresponding mass-loss rates were this time calculated for $R_{\mathrm{s}}=1.01 R_{\star}$, which is not only the most appropriate value (see, e.g. Pauldrach et al. 1986) but leads at the same time to the highest possible mass loss (see Fig. 6). We included finite disk correction in the calculations. The results can be found in Table $4^{3}$.

We compare our maximum mass-loss rates for non-rotating stars $\left(v_{\text {eq }}=0\right)$ with those predicted for two other treatments:

1) Classical $C A K$ self-regulated line-driven winds without multiple scattering and not taking into account finite disk correction (second but last column of Table 3). These mass-loss rates were calculated as in Eq. (27) of Kudritzki et al. (1989). We find that $\dot{M}_{\mathrm{CAK}}^{\mathrm{eq}}$ is roughly twice as high as $\dot{M}_{\mathrm{max}}^{\mathrm{eq}}$. This is no surprise, as it is well known that the neglect of finite disk correction leads to mass-loss rates that are too high for a selfregulated wind (Kudritzki et al. 1989).

2) Line-driven winds with multiple scattering (Vink et al. 2000). In their models Vink et al. adopted a velocity law of $\beta=1$ and the observed ratio $v_{\infty} / v_{\text {esc }}=2.6$ for O-stars and 1.3 for B-stars, as has been derived from the observations (Lamers et al. 1995). They find that the inclusion of multiple scattering increases the mass-loss rates of O-stars by about a factor of 1.4-3.2. Vink et al. have shown that their predicted massloss rates agree very well with the observed ones. In our study we did not include the effect of multiple scattering. However,

\footnotetext{
3 Any additional maximum mass-loss rates for other stellar parameters are available upon request from the authors
} 
Table 3. Stellar parameters of the nine models for which we determined the maximum mass-loss rates. $\alpha$ and $k$ are taken from Pauldrach et al. (1986). The sonic radius equals 1.01 times the stellar radius. The critical velocity at the sonic point, $v_{\text {lim }}$, is expressed in km s${ }^{-1}$ while $\sigma_{\mathrm{e}}$ is expressed in $\mathrm{cm}^{2} \mathrm{~g}^{-1}$. The last two columns give the ratio of the maximum mass-loss rates for non-rotating stars that we obtain with our formalism and the values derived from respectively classical CAK theory and the mass-loss recipe provided by Vink et al. (2000).

\begin{tabular}{cccccccccc|cc}
\hline \hline Model number & $\log L_{\star} / L_{\odot}$ & $T_{\mathrm{eff}}$ & $M_{\star}\left(M_{\odot}\right)$ & $R_{\star}\left(R_{\odot}\right)$ & $\Gamma$ & $v_{\text {lim }}$ & $\alpha$ & $k$ & $\sigma_{\mathrm{e}}$ & $\dot{M}_{\mathrm{CAK}}^{\text {eq }} / \dot{M}_{\max }^{\mathrm{eq}}$ & $\dot{M}_{\mathrm{V} \text { ink }}^{\mathrm{eq}} / \dot{M}_{\max }^{\mathrm{eq}}$ \\
\hline Model 1 & 6.0 & $50000 \mathrm{~K}$ & 60 & 15 & 0.434 & 650 & 0.64 & 0.124 & 0.34 & 2.0 & 2.1 \\
Model 2 & 6.0 & $40000 \mathrm{~K}$ & 50 & 23 & 0.521 & 435 & 0.64 & 0.124 & 0.34 & 1.9 & 1.9 \\
Model 3 & 6.0 & $30000 \mathrm{~K}$ & 40 & 42 & 0.613 & 260 & 0.59 & 0.17 & 0.32 & 1.7 & 0.7 \\
Model 4 & 5.5 & $40000 \mathrm{~K}$ & 40 & 10 & 0.209 & 780 & 0.64 & 0.124 & 0.34 & 2.0 & 1.5 \\
Model 5 & 5.5 & $30000 \mathrm{~K}$ & 35 & 18 & 0.221 & 535 & 0.59 & 0.17 & 0.32 & 1.9 & 0.8 \\
Model 6 & 5.5 & $20000 \mathrm{~K}$ & 30 & 40 & 0.250 & 325 & 0.565 & 0.32 & 0.31 & 1.8 & 1.5 \\
Model 7 & 5.0 & $40000 \mathrm{~K}$ & 30 & 8 & 0.087 & 805 & 0.64 & 0.124 & 0.34 & 2.1 & 1.0 \\
Model 8 & 5.0 & $30000 \mathrm{~K}$ & 25 & 14 & 0.089 & 550 & 0.59 & 0.17 & 0.32 & 2.0 & 0.6 \\
Model 9 & 5.0 & $20000 \mathrm{~K}$ & 20 & 32 & 0.119 & 320 & 0.565 & 0.32 & 0.31 & 1.8 & 1.3 \\
\hline
\end{tabular}

Table 4. Maximum mass-loss rates (expressed in $10^{-6} M_{\odot} \mathrm{yr}^{-1}$ ) for the stellar models listed in Table 3 for $R_{\mathrm{S}}=1.01 R_{\star}$. All $v_{\max }-\mathrm{values}$ are very similar. The listed velocities are all expressed in $\mathrm{km} \mathrm{s}^{-1}$.

\begin{tabular}{|c|c|c|c|c|c|c|c|c|}
\hline$v_{\mathrm{eq}}$ & $\dot{M}_{\max }^{\mathrm{eq}}$ & $\eta_{\mathrm{eq}}$ & $v_{\mathrm{eq}}$ & $\dot{M}_{\max }^{\mathrm{eq}}$ & $\eta_{\mathrm{eq}}$ & $v_{\mathrm{eq}}$ & $\dot{M}_{\max }^{\mathrm{eq}}$ & $\eta_{\mathrm{eq}}$ \\
\hline \multicolumn{3}{|c|}{ Model 1, $v_{\max } \simeq 480 \pm 20$} & \multicolumn{3}{|c|}{ Model 4, $v_{\max } \simeq 560 \pm 20$} & \multicolumn{3}{|c|}{ Model 7, $v_{\max } \simeq 600 \pm 20$} \\
\hline 0 & 4.30 & 0.10 & 0 & 0.81 & 0.07 & 0 & 0.14 & 0.04 \\
\hline 250 & 4.80 & 0.11 & 250 & 0.87 & 0.07 & 250 & 0.15 & 0.04 \\
\hline 500 & 7.30 & 0.17 & 500 & 1.11 & 0.10 & 500 & 0.19 & 0.06 \\
\hline 650 & 9.00 & 0.21 & 750 & 1.84 & 0.16 & 750 & 0.32 & 0.09 \\
\hline \multicolumn{3}{|c|}{ Model $2, v_{\max } \simeq 340 \pm 20$} & \multicolumn{3}{|c|}{ Model 5, $v_{\max } \simeq 370 \pm 20$} & \multicolumn{3}{|c|}{ Model $8, v_{\max } \simeq 380 \pm 20$} \\
\hline 0 & 6.10 & 0.10 & 0 & 1.01 & 0.06 & 0 & 0.16 & 0.03 \\
\hline 150 & 6.60 & 0.11 & 200 & 1.13 & 0.07 & 200 & 0.18 & 0.03 \\
\hline 300 & 8.90 & 0.15 & 400 & 1.82 & 0.11 & 400 & 0.27 & 0.05 \\
\hline 400 & 12.60 & 0.21 & 500 & 2.17 & 0.13 & 500 & 0.35 & 0.06 \\
\hline \multicolumn{3}{|c|}{ Model 3, $v_{\max } \simeq 190 \pm 20$} & \multicolumn{3}{|c|}{ Model 6, $v_{\max } \simeq 220 \pm 20$} & \multicolumn{3}{|c|}{ Model $9, v_{\max } \simeq 230 \pm 20$} \\
\hline 0 & 11.80 & 0.11 & 0 & 3.40 & 0.12 & 0 & 0.53 & 0.06 \\
\hline 100 & 13.30 & 0.12 & 100 & 3.70 & 0.13 & 100 & 0.58 & 0.06 \\
\hline 200 & 21.00 & 0.19 & 200 & 5.00 & 0.18 & 200 & 0.79 & 0.09 \\
\hline 250 & 23.10 & 0.21 & 300 & 7.20 & 0.25 & 300 & 1.13 & 0.13 \\
\hline
\end{tabular}

we do not expect the inclusion of multiple scattering to increase the maximum mass-loss rate significantly, because this maximum is not determined by the efficiency, but rather the overall ability of the momentum transfer from the photons to the gas throughout the wind. The ratios of the mass-loss rate derived from the recipe provided by Vink et al. (2000) and our maximum mass-loss rate for the nine considered stellar models are listed in the last column of Table 3 . In order to make the comparison consistent, we have used $v_{\infty} / v_{\text {esc }}=2.6$ for models 1,2 , $3,4,5,7,8$ and 1.3 for models 6 and 9 in Vink et al.'s recipe. We see that the values for the mass-loss rate derived from Vink et al.'s work are generally higher, except for Models 3, 5, 8, i.e. for hot giants and supergiants. It is precisely in these phases that stars have to lose a large amount of material in a relatively short time according to evolutionary calculations.

\section{Discussion and conclusions}

In this paper we studied the maximum mass-loss rate that can be driven by radiation pressure due to spectral lines in winds of hot stars. The models include the effects of gravity, radiation pressure by spectral lines including the finite disk correction, gas pressure, photon tiring and, for rotating models, centrifugal forces in the case of angular momentum conservation. The radiative acceleration by spectral lines is described by the CAK-formalism in terms of the force multiplier parameters, $k$ and $\alpha$ (see Sect. 2). We have solved the momentum equation by integration upward from an imposed sonic point position close to the star. The weak density dependence of the ionization, described by the force multiplier parameter $\delta$ was ignored in this study; it would reduce $\dot{M}_{\max }^{\mathrm{eq}}$ only slightly.

The results of our study can be summarised as follows. (1) Line-driven wind models at the maximum mass-loss rate for stars with a moderate rotation ( $<80 \%$ of the critical velocity) have a wind velocity that does not decrease significantly with distance. Stars rotating near critical velocity (>80\%), however, have a velocity that initially increases with distance, more or less as a $\beta$-law. From a certain distance onwards, when radiation pressure is no longer efficient, the velocity decreases outwards almost ballistically, approaching zero at infinity. Such a kinked velocity law implies that the maximum velocity is reached rather close to the star, typically within a few stellar 
radii. The values of $v_{\max }$ for maximum mass-loss rates are much smaller than the values of $v_{\infty} \simeq(1-3) v_{\text {esc }}$ derived from the classical treatment of line-driven winds.

(2) The maximum mass-loss rates of non-rotating stars are a factor 1-2 lower compared to the mass-loss rates predicted for line-driven winds with multiple scattering (Vink et al. 2000), except for hot giants and supergiants. For the latter stars we find the maximum mass-loss rates to be about 1.2-1.7 times higher than those based upon multiple scattering. Since the predicted mass-loss rates with multiple scattering agree quite well with the observed values, we conclude that the same is true for the maximum mass-loss rates derived from our formalism.

(3) We have also calculated the maximum mass-loss rates of line-driven winds of rotating stars. In these calculations we have adopted a sectorial model, i.e. the wind is assumed to move outwards in a plane of constant stellar latitude. This implies that we neglect the tendency of the gas in a rotating wind to move to the equatorial plane, i.e. the effect of wind compression (Bjorkman \& Cassinelli 1993) or the tendency of the gas to move away from the equator by radiation pressure in the lateral direction, i.e. the effect of wind inhibition (Owocki et al. 1996). We also neglected rotational flattening and gravitational darkening of the star. This means that our results of the maximum equatorial mass flux of a rotating star should be compared with those of a non-rotating star with the same values of $T_{\text {eff }}$ and $\log g$ as in the equator of the rapidly rotating star. We find that the equatorial mass-loss rate, defined as $\dot{M}_{\mathrm{eq}}=4 \pi R_{\star}^{2} F_{m}^{\mathrm{eq}}$, where $F_{m}^{\mathrm{eq}}$ is the mass flux at the equator, increases with increasing rotational velocity and reaches a maximum when $v_{\text {eq }}$ approaches $v_{\text {crit }}$. The effect of rotation will be described in detail in Aerts et al. (in preparation).

Our findings imply that, if additional effects play a role in setting the mass loss (e.g. pulsation or other instabilities), the maximum mass-loss rates of line-driven winds can be higher than predicted for self-regulated line-driven winds. However, the maximum mass-loss rates that can be accelerated and lifted out of the potential well of the star by line driving is at most 2-3 times that of a self-regulated optically-thin line-driven wind. Our conclusion therefore is that much higher mass-loss rates, e.g. those required by stellar evolution calculations close to the $\Omega \Gamma$-limit (Heger et al. 2000; Maeder \& Meynet 2000b), can only be accelerated by either radiation pressure of optically thick continuum-driven winds (e.g. as proposed for Wolf-Rayet stars by Nugis \& Lamers 2002), or by a significant increase in, and redistribution of, the line-opacity during such evolutionary stages.

We devote a separate paper (Aerts et al., in preparation) to the detailed study of the effects of rotation on the integrated maximum mass-loss rates as derived from our formalism but including gravity darkening and the oblateness of the star.
Acknowledgements. This research was started during CA's stay as guest lecturer at the Julius Institute of the University of Utrecht in the period January - February 2001. The authors are indebted to the referee, prof. Stan Owocki, for his numerous and pertinent remarks which helped us to improve our paper. CA is grateful to I. Pelupessy for sending her one of his Mathematica codes and to prof. Norbert Langer for valuable discussions and helpful comments.

\section{References}

Abbott, D. C. 1982, ApJ, 259, 282

Bjorkman, J. E., \& Cassinelli, J. P. 1993, ApJ, 409, 429

Castor, J. I., Abbott, D. C., \& Klein, R. I. 1975, ApJ, 195, 157 (CAK)

de Koter, A., Schmutz, W., \& Lamers, H. J. G. L. M. 1993, A\&A, 277, 561

Feldmeier, A., \& Shlosman, I. 2000, ApJ, 532, L125

Feldmeier, A., \& Shlosman, I. 2002, ApJ, 564, 385

Feldmeier, A., Shlosman, I., \& Hamann, W.-R. 2002, ApJ, 566, 392

Friend, D. B., \& Abbott, D. C. 1986, ApJ, 311, 701

Gayley, K. G. 2000, ApJ, 529, 1019

Heger, A., Langer, N., \& Woosley, S. E. 2000, ApJ, 528, 368

Krtička, J., \& Kubát, J. 2000, A\&A, 359, 983

Kudritzki, R. P., Pauldrach, A., Puls, J., \& Abbott, D. C. 1989, A\&A, 219, 205

Kudritzki, R. P., \& Puls, J. 2000, ARA\&A, 38, 613

Lamers, H. J. G. L. M., \& Cassinelli, J. P. 1999, Introduction to stellar winds (Cambridge University Press)

Lamers, H. J. G. L. M., Snow, T. P., \& Lindholm, D. M. 1995, ApJ, 455,269

Maeder, A., \& Meynet, G. 2000a, ARA\&A, 38, 143

Maeder, A., \& Meynet, G. 2000b, A\&A, 361, 159

Nugis, T., \& Lamers, H. J. G. L. M. 2002, A\&A, 389, 162

Owocki, S. P. 1994, Astrophys. Space Sci., 221, 3

Owocki, S. P., Cranmer, S. R., \& Blondin, J. M. 1994, ApJ, 424, 887

Owocki, S. P., Cranmer, S. R., \& Gayley, K. G. 1996, ApJ, 472, 1150

Owocki, S. P., \& Gayley, K. G. 1997, in Luminous Blue Variables: Massive Stars in Transition, ed. A. Nota, \& H. J. G. L. M. Lamers, ASP Conf. Ser., 120, 121

Owocki, S. P., \& Puls, J. 2002, ApJ, 568, 965

Pauldrach, A. W. A., Kudritzki, R. P., Puls, J., Butler, K., \& Hunsinger, J. 1994, A\&A, 283, 525

Pauldrach, A. W. A., Puls, J., \& Kudritzki, R. P. 1986, A\&A, 164, 86

Pelupessy, I., Lamers, H. J. G. L. M., \& Vink, J. S. 2000, A\&A, 359, 695

Poe, C. H., \& Friend, D. B. 1986, ApJ, 311, 317

Poe, C. H., Owocki, S. P., \& Castor, J. I. 1990, ApJ, 358, 199

Porter, J. M., \& Skouza, B. A. 1999, A\&A, 344, 205

Prinja, R. K., Barlow, M. J., \& Howarth, I. D. 1990, ApJ, 361, 607

Vink, J. S., de Koter, A., \& Lamers, H. J. G. L. M. 2000, A\&A, 362, 295

Vink, J. S., de Koter, A., \& Lamers, H. J. G. L. M. 2001, A\&A, 369, 574

Wilson, L. A., \& Bowen, G. H. 1984, Nature, 312, 429 